\title{
Claves para la implementación de los lineamientos recursos, descripción y acceso
}

\author{
Ariel Alejandro Rodríguez García *
}

Artículo recibido:

9 de diciembre de 2011.

Artículo aceptado:

16 de abril de 2012.

\section{RESUMEN}

Con la aparición de los lineamientos para la descripción y el acceso a los recursos, el proceso de catalogación se enfrenta a un desafío distinto que se caracteriza, de principio a fin, por el uso del modelado de datos y la tecnología de la información. Con ello se reconoce que el cambio en la descripción bibliográfica era necesario, pero abandonar lo anterior de golpe no será una tarea fácil. El propósito de este trabajo es subrayar que las prácticas recomendables y los flujos de trabajo formulados a partir de la implementación del nuevo código de catalogación se comprometerán a tomar ventaja en la eficiencia y flexibilidad que se quiere obtener con los datos contenidos en un registro. Uno de los principales resultados

* Centro Universitario de Investigaciones Bibliotecológicas de la UNAM, México. ariel@cuib.unam.mx

INVESTIGACIÓN BIBLIOTECOLÓGICA, Vol. 26, Núm. 56, enero/abril, 2012, México, ISSN: 0187-358X. pp. 159-179 
de la investigación es documentar la comprensión de lo que está sucediendo alrededor del aplazamiento en la ejecución del nuevo código de catalogación.

Palabras clave: RDA; Proceso de catalogación; FRBR.

\begin{abstract}
ABSTRAC
Keys for the Implementation of the Resource, Description and Access Guidelines

Ariel Alejandro Rodríguez García

With the advent of guidelines for description and access to resources, the cataloging process faces a different challenge characterized, from beginning to end, by the use of data modeling and information technology. This recognizes that changes in bibliographic description were necessary, but a sudden abandon will not be an easy task. The purpose of this paper is to highlight that the best practices and workflows made from the implementation of new cataloging code, will commit to take advantage of the efficiency and flexibility to be obtained from the data contained in a record. One of the main results of the research is to document the understanding of what is happening around the delay in the implementation of new cataloging code.
\end{abstract}

Keywords: RDA; Cataloging Process; FRBR.

\title{
INTRODUCCIÓN
}

E n junio del 2011 se dieron a conocer los resultados de la prueba "Report and recommendations of the U.S. RDA test Coordinating Committee". En dicha prueba se convocó a un grupo de expertos en catalogación para examinar y analizar los problemas que la comunidad bibliotecológica norteamericana había expresado respecto a la viabilidad de los nuevos lineamientos Recursos, Descripción y Acceso (RDA por sus siglas en inglés). 
Las metas programadas en la prueba le permitieron al Comité Directivo Conjunto para el Desarrollo de las RDA, prestar atención a las inquietudes de la comunidad norteamericana por la adopción de un nuevo código de catalogación, percatarse de las confusiones y tinglados en la interpretación de los lineamientos, y tomar en consideración las sugerencias y las recomendaciones para generar un plan estratégico.

El Reporte ${ }^{2}$ señala que la mayoría de las metas se cumplieron, unas en mayor grado que otras. Pero las que interesa enfatizar son aquellas que se refieren al uso de una herramienta en línea, que incluyan los lineamientos en inglés de uso común, y que son fáciles de usar como fundamento metodológico $^{3}$ de trabajo y con fines de formación.

En definitiva la reflexión y crítica que se haga a este documento en ámbitos académicos regionales, por ejemplo en el caso latinoamericano, sin duda reducirán la complejidad de su interpretación y la transición hacia el entendimiento de un nuevo modelo para la práctica de la catalogación y la creación del catálogo en línea de la biblioteca.

En lo concerniente a los argumentos de este trabajo de investigación, éstos se han dividido en tres partes. La primera aborda los asuntos sobre los alcances y limitaciones que se tuvieron con el grupo de prueba en cuanto al entendimiento de los Lineamientos. La segunda parte explica, groso modo, cómo introducirse al uso de los Lineamientos de acuerdo con el RDA 0.1 Key Features $^{4}$ que permite diseñar las estructuras de metadatos para aprovechar la eficiencia y flexibilidad en la captura, almacenaje, recuperación y despliegue de los datos que hace posible la tecnología de las nuevas bases de datos. Por último, observaremos cómo los nuevos lineamientos cambian el paradigma en el modelo conceptual en la práctica y flujos del trabajo de catalogación.

\section{CONDICIONES PARA LA IMPLEMENTACIÓN DE LOS LINEAMIENTOS RDA}

Cuando nos referimos a los Lineamientos, lo hacemos tratando de contextualizar las diversas formar y maneras en que se cita y conoce el nuevo código de catalogación: Recursos, Descripción y Acceso, comúnmente aludido como

2 Report and recommendations of the U.S. RDA test Coordinating Committee: Executive summary [en línea] URL: http://www.loc.gov/bibliographic-future/rda/rda-execsummary-public13june11.pdf (Consultado: 20-06-11)

3 En el texto original de la prueba se utiliza el término berramienta de trabajo. Desde nuestro punto de vista, es una traducción literal de la palabra que en el castellano tiene un significado orientado al uso de una maquinaria Es por ese por lo que usamos el término fundamento metodológico como una posición que asume lo que se entiende como conocimiento objetivo.

4 Chris Oliver, Introducing RDA a guide to the basics, London: Facet Publishing, 2010: 6. 
RDA. Resumirlo en tres letras, como una simple abstracción, nos conduce a visualizar muy parcialmente su alcance y limitaciones.

Nuestro punto de partida en esta ocasión es entender los Lineamientos como una propuesta basada en dos grandes corrientes desarrolladas en la teoría de la catalogación. ${ }^{5}$ Una de ellas se conoce como las tradiciones bibliográficas y, la otra, como una innovación dirigida con el enfoque sistémico. Por ello los Lineamientos se explican incorporando todos los medios a su alcance para responder a las exigencias de la tecnología de la información y los postulados de las Sociedades de la Información y el Conocimiento.

No es necesario detallar lo acontecido en las últimas cinco décadas en la catalogación; ${ }^{6}$ pero sí resaltar que varios hechos nos permiten confirmar el por qué de los cambios a la normativa de la descripción bibliográfica para el siglo XXI. Por ejemplo las discusiones que se dieron en torno a la creación de los Principios Internacionales de Catalogación, ${ }^{7-8}$ conocidos como los Principios de París (1961) y que se actualizaron en el año 2009, con base en los alcances y objetivos del modelo conceptual de los Requerimientos Funcionales.

Por lo que respecta a las Normas Internacionales de Descripción Bibliográfica ${ }^{9}$ (ISBD, siglas en inglés), se estableció el modelo de descripción que serviría de base para la crear los códigos de catalogación, como son las reglas de catalogación Angloamericanas y la idea del intercambio de información por medio de los formatos bibliográficos como el Formato MARC. ${ }^{10}$

Retomando la idea del nuevo código de catalogación y el uso de las tecnologías de la información, resulta casi inevitable no contextualizar los cambios en

5 Ariel Alejandro Rodríguez García. "Los objetos bibliográficos confirmados en la integración compleja de la descripción y el acceso a recursos". Investigación Bibliotecológica. Archivonomía, bibliotecología e información. Vol. 23, núm. 48 (mayo-agosto, 2009): 38.

6 Los siguientes serían algunos de los acontecimientos: Seminar on Bibliographic Records Proceeding of the Seminar held in Stockholm, 15-16 august 1990. edited by Ross Bourne Münche: K G Saur, 1992; IFLA Study Group Functional requirements for bibliographic records: final reports. München: K G Saur, 1998.

7 John D. Byrum. The birth and re-birth of the ISBDs: process and procedures for creating and revising the International Standard Bibliographic Descriptions. In: 66th IFLA Council and General Conference, Jerusalem, Israel, 13-18 August [2000] [en línea]. [S. 1.]: IFLA, 2000 [citado 2 November 2000]. Disponible en W3: <http://www.IFLA.org/IV/IFLA66/papers/118-164e. htm>

8 Mauro Guerrini. "In praise of the un-finished: the IFLA statement of international cataloguing principles (2009)." Cataloging \& Classification Quarterly. Vol. 47, no. 8: 724.; Carlo Bianchini y Mauro Guerrini. "Quis custodiet ipsos custodies? Observaciones sobre la relación entre FRBR, ICP, ISBD y RDA." Anales de documentación. No. 12, 2009: 323

9 Elena Escolano Rodríguez, Lynne Howarth, Mirna Willr, Boris Bosancie, "Novedades de ISBD. IFLA world library and information congress", 75th IFLA General Conference and Assembly, Milan, Italy, 2009.

10 Glenn Patton and Paul J. Weiss, "Cataloging after format integration", en Format integration and its effect on cataloging, training, and systems, ed. Karen Coyle (Chicago: American Library Association, 1993). 
el catálogo de la biblioteca, ${ }^{11}$ pues de ser un catálogo en tarjetas que respondía a las necesidades de los usuarios de colecciones de impresos, ha pasado a ser un catálogo electrónico y luego en línea que responde tanto a los formatos impresos como a los no impresos, y se ha transformado en un medio más versátil, flexible y funcional para ofrecer servicios que no requieren de la visita presencial en la biblioteca. Ante estas situaciones de cambio competitivo es difícil que los esquemas creados en otras épocas siguieran vigentes, por lo que la idea de los Lineamientos basados en la tecnología toma mayor fuerza.

Abundando en la explicación, los Lineamientos RDA conllevan una serie de acciones encaminadas no sólo a reconocer que era necesario el cambio sino habría que abandonar de golpe las distintas prácticas y propuestas educativas, no será tarea fácil. Evidentemente que en el ámbito de la formación profesional, por ejemplo, de los países receptores será una tarea que preocupará tanto a los actores como a las autoridades administrativas y docentes.

Hoy en día no basta con hablar de los "Lineamientos" como de un tema en boga, es necesario decir qué y cómo podemos modificar la práctica de la catalogación. Es decir, hace falta aclarar el contexto de origen, la teorización y la aplicación de ellos. En verdad nos enfrentamos a una postura distinta que puede caracterizarse genéricamente como sistémica que indaga e interviene no sólo en el ámbito de la catalogación, sino también en el modelado de datos y el uso de las tecnologías de la información.

En sus inicios, tales lineamientos surgieron como una propuesta que se observaba como opositora a las limitantes del modelo conceptual de tres entidades, ${ }^{12}$ que respaldaba a las Reglas de Catalogación Angloamericanas, segunda edición.

Hay que tener en cuenta que en ese modelo es posible apreciar una gran cantidad de elementos que les permitieron a diversos teóricos ${ }^{13}$ y grupos de investigación, reformular su estructura para adquirir conocimientos y reflexionar sí era el momento adecuado de hacerle cambios al elemento fundamental de la catalogación; el registro bibliográfico. Así es como se destaca la convicción de que la teoría de la catalogación se construye activamente por la aparición y transformación de las entidades que cohabitan en el universo bibliográfico. ${ }^{14}$

11 Arlene Taylor, Daniel N. Joudrey, The organization of information, Third edition, Westport, Connecticut, 2009, 49.

12 Allyson Carlyle, "Understanding FRBR as a conceptual model: FRBR and the bibliographic universe”, en LRTS, Vol. 5, núm. 4:267-268.

13 Richard Smiraglia, "The progress of theory in knowledge organization", Library trends, Vol. 50, núm. 3, (winter, 2002): 331-332.

14 Bárbara Tillett,"A taxonomy of bibliographic relationships”, en LRTS, Núm.32 (2). 150. 
Algunos autores se centran en hacer señalamientos sobre lo que es fundamental y lo básico para entender los Lineamientos. ${ }^{15}$ Por ejemplo, toman como punto de partida la revisión general del Programa del Control Bibliográfico; qué se entiende por registro bibliográfico en el contexto del modelado de bases de datos entidad-relación; y analizan los informes de la IFLA sobre los modelos conceptuales FRBR, entre otras cosas más.

Pero para otros, el foco de interés se ubica en el desarrollo de una nueva práctica de catalogación basada en los fundamentos de la actividad de los metadatos ${ }^{16}$ y el establecimiento de un marco lógico que ayude a la comprensión y posterior desarrollo de conveniencias para la descripción bibliográfica.

A propósito, se ha comentado que los fundamentos de la práctica de la catalogación de 1960 al 2000 se transformaron considerablemente, no sólo por la rápida proliferación de nuevos tipos de publicación, nuevas formas de contenido y nuevos formatos, sino también porque la automatización de las bibliotecas, el desarrollo de las utilería bibliográfica, el uso de la tecnología de la información y el actual movimiento de las redes en línea han cambiado el rumbo de la biblioteca y el trabajo con sus usuarios. De ahí que los Lineamientos RDA estén pensados para un entorno digital.

Retomando los resultados de la prueba del $2010^{17}$ en la meta que se refiere a "ser fácil y eficiente al usar, tanto como fundamento metodológico, como con fines de formación" se advierte que ésta no se cumplió porque hay pocos beneficios para quien adopte RDA, ya que no generará ahorros en los costos de creación de metadatos; al contrario habrá incrementos en los costos, en la formación y en la conversión de datos legados por las reglas de catalogación. De modo que el Comité Conjunto recomienda no proceder a la aplicación de los Lineamientos antes de enero de 2013.

A propósito de este señalamiento, la Biblioteca del Congreso refiere que el costo para desarrollar cursos de capacitación para RDA está implícito en los salarios de las personas, quienes se enrolan en la preparación y revisión de los materiales para la capacitación. De acuerdo con este modelo de trabajo, la Biblioteca del Congreso formula que los cursos deberían llevarse a cabo con base en los siguientes tópicos:

- RDA para el grupo de LC que crea o modifica registros bibliográficos y de autoridad;

15 Ariel Alejandro Rodríguez García, "Elementos para reflexionar sobre el nuevo estándar para la descripción y acceso a recursos", en: Biblioteca Universitaria. Revista Biblioteca Universitaria. Vol. 13, núm. 1, (junio de 2010): 58. 
- Codificación MARC con RDA para el grupo que crea o modifica registros;

- $\quad$ RDA y MARC para el grupo de LC que usa los registros.

De la American Library Association la división de la Association for Library and Technical Services (ALCTS) no tiene planes de crear algún material de entrenamiento sobre RDA, además de aquellos que se generan por medio de la serie de Webinar. De igual modo sucede con OCLC pero próximamente, no señalan el momento, ésta organizará trabajos con organismos similares para ofrecer varias sesiones de entrenamiento a través de su portal.

Para la elaboración de un curso relacionado con la enseñanza de RDA, existen cuatro constantes sobre las que es necesario reflexionar:

- Costo del material para la capacitación. Éste deberá crearse para todos los participantes, y pensarse que su acceso deberá ser gratuito y de libre acceso.

- Costo de la actualización. Éste deberá existir, como se hace en Estados Unidos, en diversos niveles: nacional, por consorcio y local.

- Tiempo para el entrenamiento. Puede variar de institución a institución, pero en promedio se solicita que sea de 20 a 40 horas.

- Curva de aprendizaje. Aquí se hace el señalamiento respecto a ¿cuánto tiempo será el necesario para que un grupo de trabajo produzca un registro RDA de manera aceptable?

Puede observarse que las recomendaciones anteriormente señaladas son congruentes con la necesidad de conocer los Lineamientos y de conducir un análisis de la práctica real, tanto del capacitador como del docente, así como de los diversos actores que permitirán la producción de materiales didácticos. Otro aspecto trascendental de los resultados que arrojó la prueba, es aquel en donde el ejercicio de la reflexión docente invita a puntualizar aspectos como el ámbito práctico-metodológico de la creación y modificación de los registros bibliográficos y de autoridad; la validez de los contenidos para quienes crean y usan los registros, y la difusión de los Lineamientos en comunidades distintas a las bibliotecológicas.

En suma en este apartado se argumentó que hasta el momento, los programas de capacitación y actualización profesional están dirigiéndose, desde nuestro punto de vista, más a la divulgación que a la manera en qué influirá en los cambios que se requieren hacer en la práctica de la catalogación y los ajustes que serán necesarios en los programas de estudios en las escuelas de bibliotecología. 
En la misma línea se reflexionó sobre la capacitación para el trabajo a partir de los resultados vertidos por el Grupo Prueba, y se encontró que existen algunos cursos de entrenamiento que no responden en su totalidad a los planteamientos y alcance del nuevo código de catalogación, aunque, se hizo mención a las maneras en que se diseñan los cursos, los costos y tiempos que implicaría para una institución preparar a su personal.

Finalmente se está de acuerdo con la decisión del Comité respecto a que es irreflexiva la implementación de los Lineamientos RDA, si éstos no se redactan de forma clara y sin ambigüedades en el inglés común.

\section{INTRODUCCIÓN AL USO DE LOS LINEAMIENTOS RDA}

Si bien es ampliamente reconocido que los RDA difieren en su modelo conceptual a las reglas de catalogación angloamericanas, también es cierto que aún no se están preparados para proponer una metodológica para su enseñanza, tanto en las escuelas de bibliotecología como en la capacitación del personal y la actualización profesional.

$\mathrm{Al}$ respecto podríamos decir que el desafío actual en la formación profesional consiste en traspasar la línea delgada que separa los logros alcanzados indiscutiblemente por las tradiciones bibliográficas, la perspectiva a futuro del modelado de bases de datos, las relaciones orientadas a los usuarios y la actividad de los metadatos.

El giro que se está dando en la práctica de la catalogación es de 180 grados, debido a que estará predominando el uso de la tecnología de la información y comunicación. Sobre éste y otros aspectos más es sobre los que enfocaremos las diversas aportaciones que nos introducen al estudio, usos aplicación e impacto de los lineamientos RDA.

Estos Lineamientos postulan la existencia y prevalencia de un modelo que está alineado ${ }^{18}$ con las diversas normativas internacionales actuales que se emplean y usan para compartir los datos bibliográficos; lo que implica que tiene conceptos y un vocabulario, así como principios que están permitiendo la reorganización de la comunidad internacional de catalogación. De esta manera podría entenderse la génesis de los lineamientos RDA 0.2 Relationsbip to the other standards for resources description and Access; 0.3 Conceptual model underlying RDA y 0.4 Objectives and principles governing resource description and access, los cuales resumen los diversos alcances y objetivos de las normativas bibliográficas creadas antes del inicio del Siglo XXI. 
Parece arriesgado decir lo anterior, pero bastaría con mencionar que quienes fueron formados con el esquema de área, elemento y puntuación descrita, ${ }^{19}$ se darán cuenta de que el registro bibliográfico permite observar las características del objeto físico y de la facilidad que se obtiene para intercambiar información bibliográfica. De acuerdo con las ISBD y las reglas de catalogación, se logró establecer la uniformidad en las bases de datos bibliográficas con base en formatos legibles por máquina.

Recordemos específicamente que en el momento en que los formatos de integración ${ }^{20}$ se introducen en la catalogación, por los años sesenta, las decisiones del catalogador se sustentaban en la composición de un registro descriptivo del ítem y en la asignación de etiquetas de acuerdo al MARC que empleaba. Es por eso por lo que actualmente se dice que el registro bibliográfico es de tipo monolítico.

En el contexto de los Lineamientos, ${ }^{21}$ sus modelos conceptuales nos permiten agudizar los sentidos en dos ejes que los sustentan, los principios sistémicos y el uso de la tecnología. Estas dos fortalezas anuncian que el registro bibliográfico actual es desarrollado con base en el Formato MARC21 y/o alguna estructura de metadatos, por lo cual es válido analizarlos y aprobarlos con base en lo que señala el principio de la representación ${ }^{22}$ (RDA 0.4.3.4 Representation) el cual señala que la descripción y las formas controladas de los nombres deben basarse en la manera en que esas entidades se describen a sí mismas.

Si hablamos del principio de representación tenemos que hablar, ante todo, de las aportaciones que hicieron Lubetzky ${ }^{23}$ y Svenonius ${ }^{24}$ para que dicho principio quedara plasmado en los objetivos generales de los Principios Internacional de Catalogación de 2009 y en el lineamiento RDA 0.4 Objetives and principles governing resources description and access.

En palabras de Lubetzky ${ }^{25}$ se menciona que la formulación de principios es una base necesaria para guiar y darle coherencia y lógica a la construcción del código de catalogación, así como proporcionarle al catalogador lineamientos

19 Barbara B. Tillett, "Bibliographic structures: the evolution of catalog entries, references, and tracings", en The conceptual foundation of descriptive cataloging, ed. Elaine Svenonius, (San Diego: Academic Press, 1989); Michael Gorman, ¿Metadatos o catalogación? Un cuestionamiento erróneo, en: Internet, metadatos y acceso a la información en bibliotecas y redes en la era electrónica, comp. Filiberto Felipe Martínez Arellano, Lina Escalona Ríos, (México: CUIB; Infoconsultores, 2000).

20 Glenn Patton and Paul J. Weiss, op cit. 11.

21 Chris Oliver, op cit. 6-8.

22 Joint Steering Committee for Development of RDA, Resources description \& Access: RDA, (Chicago: American Library Association, 2010):0-5.

23 Seymour Lubetzky, "Principles of cataloging. Final report. Phase I: descriptive cataloging", en Seymour Lubetzky: writings on the classical art of cataloging, comp. and ed Elaine Svenonius, Dorothy McGarry, (Englewood, Colorado: Libraries Unlimited, 2001), 274-277

24 Elaine Svenonius, The intellectual foundation of information organization, (Cambridge, Massachusetts: The MIT Press, 2000), 68, 71.

25 Seymour Lubetzky, op cit. 
generales que le permitan resolver los diversos casos en los cuales no exista una regla específica.

Por su parte, Svenonius ${ }^{26}$ menciona que la palabra principios ha tenido diversos usos para definir los objetivos de un sistema bibliográfico, las reglas generales de un código de catalogación y las directrices que orientan la construcción de un lenguaje bibliográfico. Aclara que los principios bibliográficos difieren de los objetivos y reglas de catalogación.

- El Principio de la representación establece que las descripciones deberán basarse en la misma entidad de información. Un subprincipio es el “principio de la precisión”, donde las descripciones deberán ser puntuales y estar soportadas en la entidad.

- El Principio de estandarización indica que las descripciones deberán ser normalizadas con toda su extensión y nivel posibles, y

- El Principio de integración subraya las descripciones para todo tipo de materiales, mismas que deberán basarse en un conjunto común de reglas que pueden ser extendidas.

Los planteamientos anteriores nos invitan a que pensemos por qué se introdujo el lineamiento RDA 0.4 Objectives and principles governing resource description and access, pues de no hacerlo podrían perderse de vista los siguientes cinco campos de acción que introduce el avance tecnológico en el código de catalogación:

a) el reemplazamiento de la rigidez por lineamientos teóricos;

b) la orientación que se busca atender respecto a las tareas del usuario;

c) su propuesta básica de clarificar el propósito de la información y los datos bibliográficos;

d) su idea de emplear diversas estructuras de metadatos;

e) el aclarar la naturaleza de las relaciones que existen entre las entidades bibliográficas y sus creadores.

Profundizar en cada uno de eso campos de acción será complejo y nos llevaría a los señalamientos que se hacen en el lineamiento RDA $0.1 \mathrm{Key}$ Features, ${ }^{27}$ que dice estar diseñado para tomar ventaja en la eficiencia y flexibilidad de la captura, almacenaje, recuperación y despliegue de datos que es posible con el empleo de las tecnologías de la información y la comunicación. 
También cobra importancia lo manifestado por Tillett ${ }^{28}$ en el 2008 y Oliver en el 2010, ${ }^{29}$ quienes ubican a estos lineamientos como una propuesta en la que predominará el uso de la tecnología y permitirá reorientar, desde nuestro punto de vista, la teoría de la catalogación en tres dimensiones:

1. Dimensión teórica-explicativa. Ésta está dirigida a ampliar y perfilar el núcleo de los conocimientos teóricos con el uso de modelos conceptuales y principios que gobiernan la descripción y el acceso.

2. Dimensión tecnológica. Las actividades se dirigirán a construir y validar procedimientos, flujos de trabajo y administración de datos a través de los "Elementos Núcleo" (RDA 0.6) y de las recomendaciones para elaborar registros mínimos.

3. Dimensiones técnico-prácticas. Actividades centradas directamente en ampliar la estructura y las tareas del usuario cuando éste usa la información; es decir, se trata de entender cada una de las Secciones de los Lineamientos en función de las tareas de encontrar, identificar, seleccionar y obtener.

La mejor solución que advertimos es que los nuevos conocimientos que se adquieran sobre el uso y aplicación de los Lineamientos, nos cambien los paradigmas creados en torno al proceso de catalogación, el diseño de las bases de datos de información bibliográfica y la constitución de un nuevo catálogo de acceso en línea de la biblioteca.

En suma, la idea que se ha expuesto sobre la introducción a los lineamientos RDA está analizada desde el contexto de los objetivos y principios que gobiernan a éstos, así como las consideraciones del principio de la representación y las dimensiones que permitirán reorientar la práctica y teoría de la catalogación. De esa forma puede concebirse que el nuevo código de catalogación generaliza los objetos de investigación que pueden difundirse tanto en la enseñanza como en la práctica de la catalogación.

Se mencionaron los escenarios y las dimensiones en las que los formadores y capacitadores pueden conducirse para conseguir un marco de aprendizaje significativo, pasando desde el progreso teórico de la catalogación hasta la manera en que deberán trabajarse los flujos de trabajo en la práctica de la catalogación.

28 Bárbara Tillett, RDA y la influencia del FRBR y otras iniciativas de la IFLA, en Memoria del Segundo Encuentro Internacional de Catalogación: tendencias en la teoría y práctica de la catalogación bibliográfica, comp. Filiberto Felipe Martínez y Arellano, Ariel Alejandro Rodríguez García (México: UNAM, CUIB; IIB; Library Outsourcing Services, 2007), 3-23.

29 Chris Oliver, op cit. 4. 


\section{Consideraciones PARA LA PRÁCtica de la CATALOgación MODERna, O, LA DESCRIPCIÓN Y ACCESO A RECURSOS}

Dentro de la investigación-acción que se está llevando a cabo sobre la implementación de los RDA, se han creado diversos textos, materiales didácticos impresos y digitales, que tienen finalidades y alcances distintos, pero todos ellos coinciden en dos beneficios para la comunidad bibliotecaria internacional, los cuales son de libre acceso y gratuitos, y tienen un acercamiento inducido, sin embargo existe el problema del idioma, es decir, están escritas y desarrolladas en inglés básico.

$\mathrm{El}$ acceso sin pagar un precio por recursos en línea, lo podemos encontrar en sitios Web como el de la Biblioteca del Congreso, (http://www.loc.gov/catdir/cpso/RDAtest/rdatraining.html), quienes han consultado el sitio se habrán percatado que los contenidos y estructuras de los materiales de aprendizaje van desde lo más general, es decir, desde el qué y cómo se concibieron los lineamientos, hasta lo más concreto que es cómo y de qué forma utilizar el contenido del RDA toolkit,(http://www.rdatoolkit.org/).

En lo referente a la aproximación inducida, esto es, visto desde el enfoque constructivista, los materiales favorecen que el aprendiz manipule los contenidos de tal manera que les permita tanto a docentes como estudiantes aprender significativamente. Es decir, una vez que se apropian de los conocimientos respecto a la constitución de los RDA, tanto los docentes como los estudiantes estarán en posibilidades de tomar decisiones reflexivas referentes a la importancia que tienen los Lineamientos para replantear la enseñanza y práctica de la catalogación moderna.

La mayor preocupación en la práctica contemporánea del proceso de catalogación, desde nuestro punto de vista, está centrada en conseguir que los datos de los distintos recursos puedan ser consultados, usados y permitan el ingreso desde distintos puntos de acceso. Alcanzar este ideal se convierte, como en otras épocas, en maravillarse de las ventajas sociales y económicas que se obtienen.

Los propósitos señalados no son necesariamente excluyentes y exclusivos para la comunidad bibliotecaria internacional. Al contrario, el mismo planteamiento de los Lineamientos estimula la participación de otras comunidades como la archivística, museográfica, geográfica, entre otras más a que consideren este nuevo código como una instrumento metodológico para la descripción y acceso a los recursos de información.

$\mathrm{El}$ Report and recommendations of the U.S. RDA test Coordinating Commit$t e e^{30}$ señala que la meta relacionada con "ser útil dentro de la comunidad bibliotecológica, pero capaz de ser utilizado por otras comunidades”, no logró 
verificarse puesto que el grupo de prueba se limitó a instituciones que realizan su práctica de catalogación con base en el Formato MARC y las reglas de catalogación angloamericanas. Aunque hay comunidades como las de la Web Semántica y Dublin Core que están desarrollando aplicaciones basadas en RDA con el propósito de revisar el verdadero potencial.

Nuevamente notamos aquí que uno de los supuestos centrales de los lineamientos RDA para ser usado en comunidades distintas a la bibliotecológica no ha sido comprobado. De tal forma, el supuesto de que los Lineamientos funcionarán efectivamente en situaciones del mundo real aún está por comprobarse y las explicaciones que se den, serán hipótesis sobre su alcance internacional.

\section{Prácticas ReCOMENDABles PARA EL USO DE LOS LINEAMIENTOS RDA}

En relación con las prácticas recomendables para el uso de los lineamientos RDA, lo que a continuación señalaremos será parte de los argumentos expresados por Oliver ${ }^{31}$ y Rodríguez García. ${ }^{32}$ En dichos textos se indica que los siguientes pasos son los recomendables para que el catalogador inicie su proceso de transición, si quiere apropiarse del nuevo código de catalogación.

Son siete los desafíos que deberán enfrentar quienes deseen calificar, aprovechar e instruirse dentro de la catalogación contemporánea. Nuestro propósito es expresar el nuevo paradigma para la práctica de la catalogación:

- Familiarizarse con los principios, objetivos y modelos conceptuales. Esto es constar que la mayoría de las comunidades bibliotecológicas han entendido, asimilado y sistematizado, las propuestas y cambios que se hacen en las normas para hacer una descripción bibliográfica, a partir de los modelos conceptuales FRBR y FRAD, así como de los cambios en los Principios Internacionales de Catalogación.

- Las tareas del usuario. Sin perder de vista los sustentos teóricos de los lineamientos, existe un eje central de esto que induce a revisar constantemente las tareas de encontrar, identificar, seleccionar y obtener un elemento que defina a las distintas entidades que se encuentran en un registro bibliográfico. Es preciso decir que en cada sección de los Lineamientos, las tareas del usuario tienen una función específica.

31 Chris Oliver, op cit. 7

32 Ariel Alejandro Rodríguez García, "Elementos para reflexionar sobre el nuevo estándar para la descripción y acceso a recursos”, en Biblioteca Universitaria. Revista de la Dirección General de Bibliotecas de la UNAM. Vol. 13, núm. 1 (junio de 2010): 55-63. 
- Amplitud en la estructura. La estructura está concebida con una perspectiva técnica con gran flexibilidad para permitir la descripción de los diversos tipos de recursos, así como todos los tipos de contenidos. El foco de interés es crear una combinación de los datos actuales con los futuros tipos de recurso y su tendencia evolutiva hacia lo que es el tipo de contenido, tipo de contenedor y tipo de medio.

- La forma de publicación. Se refiere a comprender la condición en que se integra un recurso; es decir, los atributos de la unidad simple, una monografía en múltiples partes, una publicación en partes seriadas o un recurso integrante. Nótese que se comienza a hablar de la forma de publicación como algo relevante para la descripción.

- Elementos de los datos adicionales y elementos núcleo. El tipo de elementos que se vayan a emplear tiene que ver en gran medida con la interpretación que se haga del lineamiento RDA 0.6 Core Element. El sentido de la palabra "elemento" es referido a cada una de las relaciones y atributos que son identificables y definidas por los modelos conceptuales FRBR y FRAD.

- Tome lo que vea. Aquí toma relevancia el principio de representación, debido a que la descripción de los datos de un recurso deberán reflejar las formas características del propio recurso. De ahí que sea necesario atender el impacto en el contenido de diversas instrucciones de los lineamientos.

- Énfasis en las relaciones. Las relaciones son los elementos claves de los modelos conceptuales, puesto que enfatizan la importancia que puede generarse entre las entidades y los roles que se asumen con relación a las tareas del usuario. Es por eso que seis secciones de los RDA están dedicadas al registro de las relaciones.

Dentro de los estudios e investigaciones recientes en catalogación, en los dos últimos lustros se han comenzado a desarrollar diversos acercamientos conocidos como utilerías bibliográficas y programas cooperativos en beneficio de la práctica de la catalogación. Dichas líneas tienen que ver con la innovación, el rediseño y la reorganización de los Servicios Técnicos ${ }^{33}$, particularmente con los mecanismos a seguir en las prácticas comunes, pero más

33 Sheila S. Intner, Peggy Johnson, Fundamentals of technical services management, (Chicago: American Library Association, 2008); Introduction to technical services, eighth edition. G. Edward Evans, Sheila S. Intner, and Jean Weihs, (Santa Barbara, California: Libraries Unlimited, 2011); Innovative redesign and reorganization of library technical services: paths for the future and case studies, ed. Bradford Lee Eden, (Westport, Connecticut: Libraries Unlimited, 2004); Conversation with catalogers in the 21ts century, ed Elaine R. Sanchez, (Santa Barbara, California: Libraries Unlimited, 2011). 
tarde las prácticas de la catalogación se comenzaría a denominar flujos de trabajo.

Las utilerías bibliográficas, Intner y Johnson ${ }^{34}$ señalan que diversas actividades de los servicios técnicos ocurren dentro del contexto de compartir reglas, políticas y estándares de catalogación, los cuales sirven para facilitar el funcionamiento del sistema automatizado local y, así lograr que se compartan sistemas de manera nacional y regional.

Los programas de catalogación cooperativa evidentemente se dirigen al polo de sus componentes como son el Name Authority Cooperative Program (NACO), el Monographic Bibliographic Record Cooperative Program (BIBCO), el Subject Authority Cooperative Program (SACO), y el Cooperative Online Serials Program (CONSER), así como a aquellos que son definidos en los servicios técnicos en conjunción con las actividades por realizar con los editores, distribuidores y agencias que proporcionen servicios de información.

Así, el uso de estrategias para llevar a cabo las prácticas comunes en la catalogación con base en los nuevos lineamientos, está invitando a que hagamos una minuciosa revisión a las diversas maneras en que describimos nuestros documentos, y las secuencias en que realizamos el proceso en conjunción con las reglas y los estándares de descripción. Pero si hasta el momento no se ha hecho este ejercicio de planeación y determinación de los flujos de trabajo, los catalogadores se verán sorprendidos por lo complejo que será implementar los Lineamientos RDA.

Me parece que, las consideraciones que se hacen para la práctica de la catalogación moderna, o sea la implementación de los Lineamientos RDA, nos advierte que todo lo que se haga bajo el enfoque sistémico permitirá observar la descripción bibliográfica con una visión total y evitará así el estudio aislado de los elementos que han caracterizado a las múltiples soluciones ideadas en las fichas y los registros bibliográficos actuales.

\section{FLUJOS DE TRABAJO CON RDA}

Los cambios que se producirán en la práctica de la catalogación a partir de la implementación de los RDA, afectarán directa e indirectamente una serie de rutinas y a actividades que se realizan en un departamento de Servicios Técnicos, por ello es vital que hablemos de cambios en diversas direcciones. Ante 
todo, el nuevo enfoque ${ }^{35}$ rompe con el tradicional esquema de la creación de registros compactos de las características de las entidades que conforman el universo bibliográfico; donde predominaba la técnica de la descripción como una caja receptora de datos y lo importante era llenarla con las posibles respuestas que se podían ofrecer por medio de los medios de recuperación del autor, el título y los temas; y no importaba lo que ocurriera dentro de esa caja de las entidades para establecer las relaciones.

Las bases en que se sustentan los Lineamientos son: los modelos conceptuales entidad-relación ${ }^{36}$ y el enfoque de sistemas, como en reiteradas ocasiones se ha mencionado. Al lograr expresar esas bases conceptuales a nivel del flujo de trabajo estamos en posibilidades de afirmar, hipotéticamente, que puede ser utilizado en cualquier prototipo de descripción para las entidades que se encuentran en el universo bibliográfico.

A propósito de lo anterior, las descripciones bibliográficas que se hagan a partir de la aplicación del modelo, nos parece, serán complejas y llevarán al catalogador a realizar una serie de procedimientos y pasos, por ejemplo, en los escenarios de implementación de los $\mathrm{RDA}^{37}$ se ha mencionado que pueden llevarse a cabo tres tipos de escenario.

Escenario 1. Relaciones y estructura de la base de datos orientada hacia los objetos. Señala que los datos RDA se almacenarán de acuerdo con los modelos conceptuales FRBR y FRAD cumpliendo con las características que tienen las estructuras de las bases de datos orientadas hacia los objetos. Es decir, los elementos de los datos descriptivos serán recolectados en registros que paralelamente cumplan con las indicaciones de las relaciones primarias en el modelo FRBR. Aquellos datos que se usen como puntos de acceso controlados serán reunidos en registros orientados a responder a las entidades primarias del FRAD. Los elementos de los datos que sirvan para la indización como puntos de acceso (sean estos controlados o sin controlar) serán marcados como asteriscos. En consecuencia las relaciones entre las entidades primarias de FRBR se reflejarán a través de un "link" de un registro a otro.

En el segundo escenario Vinculación de los registros bibliográficos y autoridad y el tercer escenario Estructura de bases de datos con "archivos planos" (no logados). Estructura de las bases de datos que almacenen los elementos surgidos de RDA, deberán emplear las aplicaciones convencionales en

35 Shawne D. Miksa, "Resource Description and Access (RDA) and new research potentials", en Bulletin of the American Society for Information Science and Technology, Vol. 35, núm. 5 (JuneJul., 2009), 49.

36 Robert L. Maxwell. FRBR a guide for the perplexed (Chicago: American Library Association, 2008): 8-10.

37 RDA Database Implementation Scenarios, [en línea] URL: http://www.rda-jsc.org/docs/5editor 2rev.pdf (consultado 08-11-11). 
la biblioteca. En tal situación, los datos depositados en un registro bibliográfico y de autoridad y, en algunos casos los registros de los ejemplares, estarán asociados con los registros bibliográficos. Esto es, en el supuesto de que el registro bibliográfico está vinculado con un registro de autoridad habrá que revisar las asociaciones que se obtienen entre los registros bibliográficos con los de autoridad para comprender la representación de los puntos de acceso y la creación de puntos de acceso controlados.

La descripción compleja que se obtiene en estos tres escenarios está señalando que la implementación de los lineamientos RDA pueda, según pensamos, resumirse en una serie de procedimientos o pasos:

a) Establecer mecanismos de control y seguimiento

b) Determinar qué se va hacer con los datos

c) Especificar las estrategias necesarias para reconocer cada tipo de entidades y sus relaciones.

d) Seleccionar apropiadamente los atributos de las entidades para construir las relaciones que respondan a las tareas del usuario.

e) Escoger, entre cada entidad, los elementos de los datos que permitan vincular los puntos de acceso variantes y demás datos que serán usados como puntos de acceso controlados y almacenados en los registros de autoridad.

f) Evaluar la estructura de los datos, es decir, observar la forma en que los datos permiten alcanzar la eficiencia en la creación y el mantenimiento de los datos, así como su eficacia en el acceso y la navegación en la base de datos.

Es preciso hacer notar que los escenarios y pasos descritos son una sugerencia que, de seguirse, permitirá reducir tiempos y acelerar la realización de los registros bibliográficos y de autoridad con base en los RDA. El proceso de análisis que se haga, en cada biblioteca, deberá partir del supuesto de que todos los pasos están interrelacionados; un paso, incluye al otro, y todos interactúan para la creación de la base de datos orientada a objetos. De este modo la retroalimentación será necesaria para todos y cada uno de los elementos del sistema, puesto que las revisiones, modificaciones, reestructuraciones y cambios, serán necesarias para que el sistema funcione adecuadamente.

Partiendo de lo anterior, señalamos que los flujos de trabajo propuesto por el Comité Conjunto de los RDA, son procedimientos que deberán usarse de manera reflexiva y flexible para agilizar la producción de registros bibliográficos. Y comprender que esos flujos son medios para, según sea el caso, innovar o rediseñar en la catalogación moderna. 
Consideramos que el catalogador en formación y en la práctica profesional debe identificar las tres posibles actividades que le permitirán conocer los flujos de trabajo, como son las actividades colaborativas en las cuales si se emplea una misma perspectiva en la descripción se obtendrán los resultados comunes. Las actividades cooperativas serán aquellas que marquen los programas de catalogación cooperativa y los programas locales para obtener un resultado desde el punto de vista global del resultado final. Y, las actividades de coordinación que consistirán en automatizar las tareas de tal manera que le permitan economizar tiempo, recursos y hacer un seguimiento de las distintas etapas de creación de registros bibliográficos y de autoridad.

Cada una de esas actividades y su posible interacción constituyen un importante argumento para justificar el uso de los principios RDA 0.4.3 que le dan sustento a estos nuevos lineamientos. Dichos principios también son elementos centrales de los Principios Internacionales de Catalogación que logran ese ajuste que es de gran ayuda para los desarrolladores de códigos de catalogación.

En síntesis, una buena conexión entra las prácticas recomendables y los flujos de trabajo, y de éstas con la propuesta central de este trabajo, asegura una mejor comprensión y aprendizaje de lo que está sucediendo en torno al aplazamiento en la implementación de los RDA.

\section{Perspectivas de estudio de las prácticas de la CATAlogación CON BASE EN LOS RDA}

Como comentario final sobre las claves para implementar los RDA en la práctica de la catalogación, es evidente que en el enfoque sistemático es donde se encuentran los mayores desarrollos hacia el entendimiento de los modelos conceptuales que dan sustento a éstos Lineamientos, sobre todo en lo tocante a la elaboración de las diversas relaciones que pueden encontrarse en un registro bibliográfico. También es valioso no perder de vista el origen filosófico de los principios, los cuales al ser entendidos en su total extensión serán, como señala Svenonuis, reconocidos como los diseñadores de un lenguaje bibliográfico.

En lo tocante a los resultados obtenidos por el Grupo de Prueba en Estados Unidos para la implementación de los RDA, particularmente las metas relacionadas con la capacitación y la lengua de los Lineamientos, observamos importantes avances, sobre todo en la elaboración y difusión de los materiales para la capacitación, no así para la formación profesional. De ahí que los profesores de las escuelas de bibliotecología deberán elaborar proyectos para 
hacer una revisión curricular y articular los mecanismos pedagógicos y educativos inevitables que respondan a las nuevas exigencias requeridas por la nueva practica de la catalogación.

Naturalmente, los problemas que enfrentan las comunidades que desean adoptar estos Lineamientos, exigirán una competencia intelectual más compleja. De ahí, el llamamiento a crear Entornos Virtuales de Investigación a nivel regional, nacional y local para proponer los mecanismos que implican una mejor apropiación y práctica con los modelos que se proponen para la implementación de los RDA.

Podríamos concluir que el cómo y el cuándo de la implementación de los Lineamientos RDA en Latinoamérica son aspectos que dependen de la finalidad específica de cada institución educativa, de los diversos desarrollos de los sistemas bibliotecarios, del tipo de proceso comunicativo de los avances que tengan con respecto a la apropiación de los nuevos lineamientos y de las particularidades del contexto general donde esté ocurriendo algún otro acontecimiento como el que nos reunió en esta ocasión.

\section{TRABAJOS CITADOS}

Bianchini, Carlo y Mauro Guerrini, "Quis custodiet ipsos custodies? Observaciones sobre la relación entre FRBR, ICP, ISBD y RDA", en Anales de documentación (2009) Núm. 12.

Byrum, John D., "The birth and re-birth of the ISBDs: process and procedures for creating and revising the International Standard Bibliographic Descriptions", en 66th IFLA Council and General Conference, Jerusalem, Israel, 13-18 August [2000], [en línea], disponible en URL: http://www.IFLA.org/IV/IFLA66/papers/118164e.htm

Carlyle, Allyson "Understanding FRBR as a conceptual model: FRBR and the bibliographic universe." LRTS 5, núm. 4:267-268.

Coyle, Karen, ed., Format integration and its effect on cataloging, training, and systems, (Chicago: American Library Association, 1993).

Eden, Bradford Lee, ed., Innovative redesign and reorganization of $l i$ brary technical services: paths for the future and case studies, (Westport, Connecticut: Libraries Unlimited, 2004).

Escolano Rodríguez, Elena, Lynne Howarth, Mirna Willr, Boris Bosancie, "Novedades de ISBD. IFLA world library and information congress", en 75th IFLA General Conference and Assembly, Milan, Italy, 2009.

Evans, G. Edward, Sheila S. Intner, and Jean Weihs, Introduction to technical services, eighth edition, (Santa Barbara, California: Libraries Unlimited, 2011). 
Guerrini, Mauro. "In praise of the un-finished: the IFLA statement of international cataloguing principles", Cataloging E Classification Quarterly, (2009)Vol. 47, núm. 8.

IFLA Study Group, Functional requirements for bibliographic records: final reports, München: K G Saur, 1998.

International Conference on the Principles and Future Development of AARC, Principles and future of AACR, Edited by Jean Weihs, ALA, 1997.

Intner, Sheila, and Peggy Johnson, Fundamentals of technical services management, (Chicago, Illinois: ALA, 2008).

Joint Steering Committee for Development of RDA, Resources description \& Access: RDA, (Chicago: American Library Association, 2010).

Lubetzky, Seymour, "Principles of cataloging. Final report. Phase I: descriptive cataloging", en Seymour Lubetzky: writings on the classical art of cataloging, edited by Elaine Svenonius and Dorothy McGarry, 256, Englewood, Col. : Libraries Unlimited, 2001.

Martínez Arellano, Filiberto Felipe, Lina Escalona Ríos, comp., Internet, metadatos y acceso a la información en bibliotecas y redes en la era electrónica, (México: CUIB; Infoconsultores, 2000).

Martínez Arellano, Filiberto Felipe, Ariel Alejandro Rodríguez García comp., Memoria del Segundo Encuentro Internacional de Catalogación: tendencias en la teoría y práctica de la catalogación bibliográfica, (México: UNAM, CUIB; IIB; Library Outsourcing Services, 2007).

Maxwell, Robert L., FRBR a guide for the perplexed, (Chicago: American Library Association, 2008).

Miksa, Shawne D., "Resource Description and Access (RDA) and new research potentials", en Bulletin of the American Society for Information Science and Technology, Vol. 35, núm. 5 (June-Jul., 2009).

Oliver, Chris, Introduccing RDA: a guide to the basics, London: Facet Publishing, 2010.

RDA Database Implementation Scenarios [en línea], URL: http:// www.rda-jsc.org/docs/5editor2rev.pdf, (consultado 08-11-11).

Report and recommendations of the U.S. RDA test Coordinating Committee [en línea], URL: http://www.loc.gov/bibliographic-future/ rda/ (Consultado: 01-07-2011)

Report and recommendations of the U.S. RDA test Coordinating Committee: Executive summary [en línea], URL: http://www.loc.gov/ bibliographic-future/rda/rda-execsummary-public-13june11.pdf, (consultado: 20-06-11).

Rodríguez García, Ariel Alejandro, "Elementos para reflexionar sobre el nuevo estándar para la descripción y acceso a recursos", en Biblioteca Universitaria. Revista Biblioteca Universitaria. Vol. 13, núm. 1, (junio de 2010). 
"Los estudios de caso en la catalogación: sus contextos teórico-prácticos", en Investigación Bibliotecológica, Archivonomía, Bibliotecología e Información 25, núm. 53, (2011): 185-200.

"Los objetos bibliográficos confirmados en la integración compleja de la descripción y acceso a recursos", en Investigación Bibliotecológica, Archivonomía, Bibliotecología e Información, 23, núm. 48 (mayo-agosto 2009): 33-59.

Sanchez, Elaine R. ed., Conversation with catalogers in the 21ts century, (Santa Barbara, California: Libraries Unlimited, 2011).

Seminar on Bibliographic Records, Proceeding of the Seminar beld in Stockholm, 15-16 august 1990, edited by Ross Bourne Münche: K G Saur, 1992.

Smiralgia, Richard P, "The progress of theory in knowledge organization", en Library trends 50, núm. 3, (2002): 330-349.

Svenonius, Elaine, The intellectual foundation of information organization, (Chicago: The MIT Press, 2000).

Svenonius, Elaine, ed., The conceptual foundation of descriptive cataloging, (San Diego: Academic Press, 1989).

Svenonius, Elaine, Dorothy McGarry comp. and ed., Seymour Lubetzky: writings on the classical art of cataloging, (Englewood, Colorado: Libraries Unlimited, 2001).

Taylor, Arlene, Daniel N. Joudrey, The organization of information, Third edition, (Westport, Connecticut: Libraries Unlimited, 2009).

Tillet, Barbara, "A taxonomy of bibliographic relationships", LRST 32, núm. 2, 150.

, ¿Qué es FRBR? un modelo conceptual del universo bibliográfico, Washington: The Library of Congress, 2004.

Vellucci, Sherry L., "Bibliographic relationships", International Conference on the Principles and Future Development of AACR, Chicago: American Library Association, 1998, 105-128. 
